PROCEEDINGS OF THE

AMERICAN MATHEMATICAL SOCIETY

Volume 127, Number 9, Pages 2703-2713

S 0002-9939(99)04840-6

Article electronically published on April 15, 1999

\title{
RELATIVE MODULAR THEORY FOR A WEIGHT
}

\author{
HIDEAKI IZUMI
}

(Communicated by David R. Larson)

\begin{abstract}
We consider the balanced weight $\chi$ of a semi-finite weight $\varphi$ and a (not necessarily faithful) normal positive functional $\psi$ on a von Neumann algebra $\mathcal{M}$, and discuss how the modular operator $\Delta_{\chi}$ and the modular conjugation $J_{\chi}$ are described under the identification of the standard Hilbert space $\mathcal{H}_{\chi}$ with $\mathcal{H}_{\varphi} \oplus p \mathcal{H}_{\varphi} \oplus p^{\prime} \mathcal{H}_{\varphi} \oplus p p^{\prime} \mathcal{H}_{\varphi}$, where $p$ is the support projection of $\psi$ and $p^{\prime}=J_{\varphi} p J_{\varphi} \in \mathcal{M}^{\prime}$.
\end{abstract}

In the theory of von Neumann algebras, relative modular operators introduced by A. Connes [2] and T. Digernes [3] play important rôles as a non-commutative analogue of Radon-Nikodym derivative. In [6], H. Kosaki extended Connes' results. He considered the balanced functional of a faithful normal positive functional and a (not necessarily faithful) normal positive functional on a ( $\sigma$-finite) von Neumann algebra $\mathcal{M}$, and by considering the modular operator $\Delta_{\chi}$, he obtained relative modular operators $\Delta_{\psi \varphi}$ and $\Delta_{\varphi \psi}$. Moreover, he showed that the modular conjugation $J_{\chi}$ can be described in terms of $J_{\varphi}$ and its restrictions. His results thus obtained is used in [7] to construct $\mathcal{M}_{*}$-valued KMS functions, which he in turn used to prove that the $L^{2}$-space $L^{2}(\mathcal{M}, \varphi)$ obtained by the complex interpolation of $\mathcal{M}$ and $\mathcal{M}_{*}$ is exactly the standard Hilbert space $\mathcal{H}_{\varphi}$.

In this paper, we will extend Kosaki's results to the weight case, namely, we consider the balanced weight $\chi$ of a faithful normal semi-finite weight $\varphi$ and a (not necessarily faithful) normal positive functional $\psi$ on a von Neumann algebra $\mathcal{M}$ and get similar results as in [6]. In particular, we determine the modular conjugation $J_{\chi}$ for the balanced weight $\chi$. These results will be used in [5] to construct KMS functions with respect to $\varphi$ and $\psi$.

First, we fix the notations in modular theory ([10], [11]).

Let $\mathcal{M}$ be a von Neumann algebra and $\varphi$ be a faithful normal semi-finite weight on $\mathcal{M}$. We set

$$
\mathfrak{n}_{\varphi}=\left\{x \in \mathcal{M} \mid \varphi\left(x^{*} x\right)<\infty\right\} .
$$

Let $\left\{\pi_{\varphi}, \mathcal{H}_{\varphi}, \Lambda_{\varphi}\right\}$ be the semi-cyclic representation induced by $\varphi, \mathcal{B}_{\varphi}=\Lambda_{\varphi}\left(\mathfrak{n}_{\varphi}\right)$ be left bounded vectors, $\pi_{l}^{\varphi}(\xi), \xi \in \mathcal{B}_{\varphi}$, be the left multiplication by $\xi$ and $\mathfrak{A}_{\varphi}=$ $\Lambda_{\varphi}\left(\mathfrak{n}_{\varphi} \cap \mathfrak{n}_{\varphi}^{*}\right)$ be the left Hilbert algebra associated to the semi-cyclic representation $\left\{\pi_{\varphi}, \mathcal{H}_{\varphi}, \Lambda_{\varphi}\right\}$. Next, we define $S_{\varphi}: \mathfrak{A}_{\varphi} \rightarrow \mathfrak{A}_{\varphi}$ by

$$
S_{\varphi} \Lambda_{\varphi}(x)=\Lambda_{\varphi}\left(x^{*}\right), x \in \mathfrak{n}_{\varphi} \cap \mathfrak{n}_{\varphi}^{*} .
$$

Received by the editors March 31, 1997 and, in revised form, November 24, 1997.

1991 Mathematics Subject Classification. Primary 46L10.

(C)1999 American Mathematical Society 
Then $S_{\varphi}$ is a conjugate-linear preclosed operator. By polar decomposition, we have

$$
\overline{S_{\varphi}}=J_{\varphi} \Delta_{\varphi}^{1 / 2},
$$

where $\overline{S_{\varphi}}$ denotes the closure of $S_{\varphi}$. The operator $J_{\varphi}$ is a conjugate-linear unitary on $\mathcal{H}_{\varphi}$ and is called the modular conjugation, while the operator $\Delta_{\varphi}$ is a non-singular positive self-adjoint operator on $\mathcal{H}_{\varphi}$ and is called the modular operator.

Next, we set

$$
\mathfrak{B}_{\varphi}^{\prime}=\left\{\eta \in \mathcal{H}_{\varphi} \mid \sup \left\{\left\|\pi_{l}^{\varphi}(\xi) \eta\right\| \mid \xi \in \mathfrak{A}_{\varphi},\|\xi\| \leq 1\right\}<+\infty\right\} .
$$

An element of $\mathfrak{B}_{\varphi}^{\prime}$ is called a right bounded vector. We write the right multiplication by $\eta \in \mathcal{B}_{\varphi}^{\prime}$ as $\pi_{r}^{\varphi}(\eta)$.

We define the right Hilbert algebra $\mathfrak{A}_{\varphi}^{\prime}$ by

$$
\mathfrak{A}_{\varphi}^{\prime}=\mathfrak{B}_{\varphi}^{\prime} \cap \mathcal{D}\left(S_{\varphi}^{*}\right) .
$$

If we write

then we have

$$
\begin{aligned}
\xi^{\sharp} & =\overline{S_{\varphi}} \xi, \quad \xi \in \mathcal{D}\left(\overline{S_{\varphi}}\right) ; \\
\eta^{b} & =S_{\varphi}^{*} \eta, \quad \eta \in \mathcal{D}\left(S_{\varphi}^{*}\right),
\end{aligned}
$$

$$
\begin{aligned}
& \pi_{l}^{\varphi}\left(\xi^{\sharp}\right)=\pi_{l}^{\varphi}(\xi)^{*}, \quad \xi \in \mathfrak{A}_{\varphi} ; \\
& \pi_{r}^{\varphi}\left(\eta^{b}\right)=\pi_{r}^{\varphi}(\eta)^{*}, \quad \eta \in \mathfrak{A}_{\varphi}^{\prime} .
\end{aligned}
$$

Moreover, we define the positive cone $\mathcal{P}_{\varphi}^{\natural}$ by

$$
\mathcal{P}_{\varphi}^{\natural}=\overline{\left\{\pi_{l}(\xi) J_{\varphi} \xi \mid \xi \in \mathfrak{A}_{\varphi}\right\}} .
$$

Since the representation $\pi_{\varphi}$ is faithful, from now on, we will identify $\pi_{\varphi}(\mathcal{M})$ by $\mathcal{M}$ and write $x$ instead of $\pi_{\varphi}(x), x \in \mathcal{M}$.

Now we fix

$$
\psi \in\left(\mathcal{M}_{*}\right)_{+} \cdot
$$

Then by [1], [4] (see also [11]), there exists a unique $\xi \in \mathcal{P}_{\varphi}^{\natural}$ such that

$$
\psi=\omega_{\xi}=(\cdot \xi \mid \xi)
$$

We remark that the support projection $p \in \mathcal{M}$ of $\psi$ is equal to the projection onto $\left[\mathcal{M}^{\prime} \xi\right]$. Here, the notation $[\mathcal{K}]$ means the closed linear span of $\mathcal{K}$.

Let

$$
\begin{aligned}
\mathcal{N} & =\mathcal{M} \otimes M_{2}(\mathcal{C}) \\
& =\left\{\left(\begin{array}{ll}
a & b \\
c & d
\end{array}\right) \mid a, b, c, d \in \mathcal{M}\right\}
\end{aligned}
$$

and let $\bar{p}$ be the projection in $\mathcal{N}$ such that

$$
\bar{p}=\left(\begin{array}{ll}
1 & 0 \\
0 & p
\end{array}\right) .
$$

We denote the reduced algebra of $\mathcal{N}$ by the projection $\bar{p}$ by $\mathcal{N}_{\bar{p}}$, that is,

$$
\mathcal{N}_{\bar{p}}=\left\{\left(\begin{array}{ll}
a & b \\
c & d
\end{array}\right) \mid a \in \mathcal{M}, b \in \mathcal{M} p, c \in p \mathcal{M}, d \in p \mathcal{M} p\right\} .
$$

Here, we note that if $\left(\begin{array}{ll}a & b \\ c & d\end{array}\right) \in \mathcal{N}_{\bar{p}}$ is positive, then $a \geq 0, d \geq 0$ and $c=b^{*}$. 
Next, we consider the balanced weight $\chi$ on $\mathcal{N}_{\bar{p}}$ determined by

$$
\chi\left(\left(\begin{array}{cc}
a & b \\
b^{*} & d
\end{array}\right)\right)=\varphi(a)+\psi(d),\left(\begin{array}{cc}
a & b \\
b^{*} & d
\end{array}\right) \in\left(\mathcal{N}_{\bar{p}}\right)_{+} .
$$

Then we have

$$
\begin{aligned}
\chi\left(\left(\begin{array}{ll}
a & b \\
c & d
\end{array}\right)^{*}\left(\begin{array}{ll}
a & b \\
c & d
\end{array}\right)\right) & =\chi\left(\left(\begin{array}{ll}
a^{*} a+c^{*} c & a^{*} b+c^{*} d \\
b^{*} a+d^{*} c & b^{*} b+d^{*} d
\end{array}\right)\right) \\
& =\varphi\left(a^{*} a+c^{*} c\right)+\psi\left(b^{*} b+d^{*} d\right) \\
& =\varphi\left(a^{*} a\right)+\varphi\left(c^{*} c\right)+\psi\left(b^{*} b\right)+\psi\left(d^{*} d\right) .
\end{aligned}
$$

Hence, by [8, 3.1], [11, Lemma VIII.3.1], we have the following.

Lemma 1. (1) The weight $\chi$ on $\mathcal{N}_{\bar{p}}$ is faithful, normal and semi-finite.

(2) $\mathfrak{n}_{\chi}=\left\{\left(\begin{array}{ll}a & b \\ c & d\end{array}\right) \in \mathcal{N}_{\bar{p}} \mid a \in \mathfrak{n}_{\varphi}, c \in p \mathfrak{n}_{\varphi}\right\}$.

We set $p^{\prime}=J_{\varphi} p J_{\varphi} \in \mathcal{M}^{\prime}$, so that $p^{\prime}$ is the projection onto $[\mathcal{M} \xi]$.

By the formula (1), for $\left(\begin{array}{ll}a & b \\ c & d\end{array}\right) \in \mathfrak{n}_{\chi}$, we have

$$
\chi\left(\left(\begin{array}{ll}
a & b \\
c & d
\end{array}\right)^{*}\left(\begin{array}{ll}
a & b \\
c & d
\end{array}\right)\right)=\left\|\Lambda_{\varphi}(a)\right\|^{2}+\left\|\Lambda_{\varphi}(c)\right\|^{2}+\|b \xi\|^{2}+\|d \xi\|^{2} .
$$

Here, we note that

$$
\begin{array}{ll}
\Lambda_{\varphi}(a) & \in \mathfrak{n}_{\varphi} \subset \mathcal{H}_{\varphi}, \\
\Lambda_{\varphi}(c) & \in p \Lambda_{\varphi}\left(\mathfrak{n}_{\varphi}\right) \subset p \mathcal{H}_{\varphi} \\
b \xi & \in \mathcal{M} \xi=\mathcal{M} \xi \subset p^{\prime} \mathcal{H}_{\varphi} \\
d \xi & \in p \mathcal{M} p \xi=p \mathcal{M} \xi \subset p p^{\prime} \mathcal{H}_{\varphi}
\end{array}
$$

Thus, the formula (1) means that the pre-Hilbert space $\mathfrak{n}_{\chi}$ equipped with the inner product induced by $\chi$ is isometrially mapped into $\mathcal{H}_{\varphi} \oplus p \mathcal{H}_{\varphi} \oplus p^{\prime} \mathcal{H}_{\varphi} \oplus p p^{\prime} \mathcal{H}_{\varphi}$ via

$$
\eta_{\chi}:\left(\begin{array}{ll}
a & b \\
c & d
\end{array}\right) \mapsto\left(\begin{array}{c}
\Lambda_{\varphi}(a) \\
\Lambda_{\varphi}(c) \\
b \xi \\
d \xi
\end{array}\right) .
$$

Since $p \Lambda_{\varphi}\left(\mathfrak{n}_{\varphi}\right) \subset p \mathcal{H}_{\varphi}, \mathcal{M} \xi \subset p^{\prime} \mathcal{H}_{\varphi}$ and $p \mathcal{M} \xi \subset p p^{\prime} \mathcal{H}_{\varphi}$, the image of $\eta_{\chi}$ is dense in $\mathcal{H}_{\varphi} \oplus p \mathcal{H}_{\varphi} \oplus p^{\prime} \mathcal{H}_{\varphi} \oplus p p^{\prime} \mathcal{H}_{\varphi}$. Hence the Hilbert space $\mathcal{H}_{\chi}$, the completion of $\mathfrak{n}_{\chi}$, can be identified with $\mathcal{H}_{\varphi} \oplus p \mathcal{H}_{\varphi} \oplus p^{\prime} \mathcal{H}_{\varphi} \oplus p p^{\prime} \mathcal{H}_{\varphi}$.

Now, we will examine how the representation $\pi_{\chi}$ of $\mathcal{N}_{\bar{p}}$ is described under this identification.

For

$$
\left(\begin{array}{ll}
a & b \\
c & d
\end{array}\right) \in \mathcal{N}_{\bar{p}} \text { and }\left(\begin{array}{ll}
e & f \\
g & h
\end{array}\right) \in \mathfrak{n}_{\chi}
$$


we have

$$
\begin{aligned}
& \pi_{\chi}\left(\left(\begin{array}{ll}
a & b \\
c & d
\end{array}\right)\right) \eta_{\chi}\left(\left(\begin{array}{ll}
e & f \\
g & h
\end{array}\right)\right)=\eta_{\chi}\left(\left(\begin{array}{ll}
a & b \\
c & d
\end{array}\right)\left(\begin{array}{ll}
e & f \\
g & h
\end{array}\right)\right) \\
& =\eta_{\chi}\left(\left(\begin{array}{ll}
a e+b g & a f+b h \\
c e+d g & c f+d h
\end{array}\right)\right) \\
& =\left(\begin{array}{c}
\Lambda_{\varphi}(a e+b g) \\
\Lambda_{\varphi}(c e+d g) \\
(a f+b h) \xi \\
(c f+d h) \xi
\end{array}\right) \\
& =\left(\begin{array}{cccc}
a & \left.b\right|_{p \mathcal{H}_{\varphi}} & 0 & 0 \\
c & \left.d\right|_{p \mathcal{H}_{\varphi}} & 0 & 0 \\
0 & 0 & \left.a\right|_{p^{\prime} \mathcal{H}_{\varphi}} & \left.b\right|_{p p^{\prime} \mathcal{H}_{\varphi}} \\
0 & 0 & \left.c\right|_{p^{\prime} \mathcal{H}_{\varphi}} & \left.d\right|_{p p^{\prime} \mathcal{H}_{\varphi}}
\end{array}\right)\left(\begin{array}{c}
\Lambda_{\varphi}(e) \\
\Lambda_{\varphi}(g) \\
f \xi \\
h \xi
\end{array}\right) .
\end{aligned}
$$

By the identification of $\mathcal{H}_{\chi}$ with $\mathcal{H}_{\varphi} \oplus p \mathcal{H}_{\varphi} \oplus p^{\prime} \mathcal{H}_{\varphi} \oplus p p^{\prime} \mathcal{H}_{\varphi}$ via $\eta_{\chi}$, we conclude that the representation $\pi_{\chi}$ is described as

$$
\pi_{\chi}\left(\left(\begin{array}{ll}
a & b \\
c & d
\end{array}\right)\right)=\left(\begin{array}{cccc}
a & \left.b\right|_{p \mathcal{H}_{\varphi}} & 0 & 0 \\
c & \left.d\right|_{p \mathcal{H}_{\varphi}} & 0 & 0 \\
0 & 0 & \left.a\right|_{p^{\prime} \mathcal{H}_{\varphi}} & \left.b\right|_{p p^{\prime} \mathcal{H}_{\varphi}} \\
0 & 0 & \left.c\right|_{p^{\prime} \mathcal{H}_{\varphi}} & \left.d\right|_{p p^{\prime} \mathcal{H}_{\varphi}}
\end{array}\right) \in \mathcal{L}\left(\mathcal{H}_{\varphi} \oplus p \mathcal{H}_{\varphi} \oplus p^{\prime} \mathcal{H}_{\varphi} \oplus p p^{\prime} \mathcal{H}_{\varphi}\right) .
$$

Since the representation $\pi_{\chi}$ is faithful, we identify $\pi_{\chi}\left(\mathcal{N}_{\bar{p}}\right)$ with $\mathcal{N}_{\bar{p}}$.

We define

$$
S_{\chi}\left(\left(\begin{array}{c}
\Lambda_{\varphi}(a) \\
\Lambda_{\varphi}(c) \\
b \xi \\
d \xi
\end{array}\right)\right)=\left(\begin{array}{c}
\Lambda_{\varphi}\left(a^{*}\right) \\
b^{*} \xi \\
\Lambda_{\varphi}\left(c^{*}\right) \xi \\
d^{*} \xi
\end{array}\right),\left(\begin{array}{c}
\Lambda_{\varphi}(a) \\
\Lambda_{\varphi}(c) \\
b \xi \\
d \xi
\end{array}\right) \in \mathfrak{n}_{\chi} \cap \mathfrak{n}_{\chi}^{*}
$$

It is easy to see that $S_{\chi}$ is well-defined. Then, by (1) we have

\section{Lemma 2.}

$$
\left(\begin{array}{c}
\Lambda_{\varphi}(a) \\
\Lambda_{\varphi}(c) \\
b \xi \\
d \xi
\end{array}\right) \in \mathfrak{A}_{\chi}=\Lambda_{\chi}\left(\mathfrak{n}_{\chi} \cap \mathfrak{n}_{\chi}^{*}\right)
$$

if and only if

$$
a \in \mathfrak{n}_{\varphi} \cap \mathfrak{n}_{\varphi}^{*}, b \in \mathfrak{n}_{\varphi}^{*} p, c \in p \mathfrak{n}_{\varphi} .
$$

Note that the densely-defined conjugate-linear operator $S_{\chi}$ is closable. By looking at each component, we have

$$
\begin{array}{lclll}
(1) & S_{\varphi}: & \Lambda_{\varphi}(a) \in \mathfrak{A}_{\chi} & \mapsto & \Lambda_{\varphi}\left(a^{*}\right) \in \mathfrak{A}_{\chi}, \\
(2) & S_{\psi \varphi}: & \Lambda_{\varphi}(c) \in p \Lambda_{\varphi}\left(\mathfrak{n}_{\varphi}\right) & \mapsto & c^{*} \xi \in \mathfrak{n}_{\varphi}^{*} \xi, \\
(3) & S_{\varphi \psi}: \quad b \xi \in \mathfrak{n}_{\varphi}^{*} \xi & \mapsto & \Lambda_{\varphi}\left(b^{*}\right) \in p \Lambda_{\varphi}\left(\mathfrak{n}_{\varphi}\right), \\
(4) & S_{\psi}: & d \xi \in p \mathcal{M} \xi & \mapsto & d^{*} \xi \in p \mathcal{M} \xi
\end{array}
$$


Then it is easily seen that

$$
S_{\chi}=\left(\begin{array}{cccc}
S_{\varphi} & 0 & 0 & 0 \\
0 & 0 & S_{\varphi \psi} & 0 \\
0 & S_{\psi \varphi} & 0 & 0 \\
0 & 0 & 0 & S_{\psi}
\end{array}\right) .
$$

Since $S_{\chi}$ is densely defined and closable, we conclude that

(a) $S_{\psi \varphi}$ is a densely-defined closable operator from $p \mathcal{H}_{\varphi}$ to $p^{\prime} \mathcal{H}_{\varphi}$;

(b) $S_{\varphi \psi}$ is a densely-defined closable operator from $p^{\prime} \mathcal{H}_{\varphi}$ to $p \mathcal{H}_{\varphi}$;

(c) $S_{\psi}$ is a densely-defined closable operator on $p p^{\prime} \mathcal{H}_{\varphi}$.

Furthermore, we have

$$
\begin{aligned}
& S_{\chi}^{*} \overline{S_{\chi}}=\left(\begin{array}{cccc}
S_{\varphi} & 0 & 0 & 0 \\
0 & 0 & S_{\varphi \psi} & 0 \\
0 & S_{\psi \varphi} & 0 & 0 \\
0 & 0 & 0 & S_{\psi}
\end{array}\right)^{*} \overline{\left(\begin{array}{cccc}
S_{\varphi} & 0 & 0 & 0 \\
0 & 0 & S_{\varphi \psi} & 0 \\
0 & S_{\psi \varphi} & 0 & 0 \\
0 & 0 & 0 & S_{\psi}
\end{array}\right)} \\
& =\left(\begin{array}{cccc}
S_{\varphi}^{*} & 0 & 0 & 0 \\
0 & 0 & S_{\psi \varphi}^{*} & 0 \\
0 & S_{\varphi \psi}^{*} & 0 & 0 \\
0 & 0 & 0 & S_{\psi}^{*}
\end{array}\right)\left(\begin{array}{cccc}
\overline{S_{\varphi}} & 0 & 0 & 0 \\
0 & 0 & \overline{S_{\varphi \psi}} & 0 \\
0 & \overline{S_{\psi \varphi}} & 0 & 0 \\
0 & 0 & 0 & \overline{S_{\psi}}
\end{array}\right) \\
& =\left(\begin{array}{cccc}
S_{\varphi}^{*} \overline{S_{\varphi}} & 0 & 0 & 0 \\
0 & S_{\psi \varphi}^{*} \overline{S_{\psi \varphi}} & 0 & 0 \\
0 & 0 & S_{\varphi \psi}^{*} \overline{S_{\varphi \psi}} & 0 \\
0 & 0 & 0 & S_{\psi}^{*} \overline{S_{\psi}}
\end{array}\right) .
\end{aligned}
$$

We define

$$
\begin{array}{ll}
\Delta_{\psi \varphi}=S_{\psi \varphi}^{*} \overline{S_{\psi \varphi}} & \text { on } p \mathcal{H}_{\varphi}, \\
\Delta_{\varphi \psi}=S_{\varphi \psi}^{*} \overline{S_{\varphi \psi}} & \text { on } p^{\prime} \mathcal{H}_{\varphi}, \\
\Delta_{\psi}=S_{\psi}^{*} S_{\psi} & \text { on } p p^{\prime} \mathcal{H}_{\varphi} .
\end{array}
$$

These are all non-singular positive self-adjoint operators. Among them, $\Delta_{\psi \varphi}$ and $\Delta_{\varphi \psi}$ are called relative modular operators. The above calculation shows that

$$
\Delta_{\chi}=\left(\begin{array}{cccc}
\Delta_{\varphi} & 0 & 0 & 0 \\
0 & \Delta_{\psi \varphi} & 0 & 0 \\
0 & 0 & \Delta_{\varphi \psi} & 0 \\
0 & 0 & 0 & \Delta_{\psi}
\end{array}\right)
$$

Next, we will determine the modular conjugation $J_{\chi}$. To do this, it is important to consider the commutant $\left(\mathcal{N}_{\bar{p}}\right)^{\prime}$ of $\mathcal{N}_{\bar{p}}$ acting on $\mathcal{H}_{\varphi} \oplus p \mathcal{H}_{\varphi} \oplus p^{\prime} \mathcal{H}_{\varphi} \oplus p p^{\prime} \mathcal{H}_{\varphi}$.

Lemma 3. The commutant $\mathcal{N}_{\bar{p}}^{\prime}$ is given by

$$
\mathcal{N}_{\bar{p}}^{\prime}=\left\{\left(\begin{array}{cccc}
a^{\prime} & 0 & \left.b^{\prime}\right|_{p^{\prime} \mathcal{H}_{\varphi}} & 0 \\
0 & \left.a^{\prime}\right|_{p \mathcal{H}_{\varphi}} & 0 & \left.b^{\prime}\right|_{p p^{\prime} \mathcal{H}_{\varphi}} \\
p^{\prime} c^{\prime} & 0 & \left.p^{\prime} d^{\prime}\right|_{p^{\prime} \mathcal{H}_{\varphi}} & 0 \\
0 & \left.p^{\prime} c^{\prime}\right|_{p \mathcal{H}_{\varphi}} & 0 & \left.p^{\prime} d^{\prime}\right|_{p^{\prime} \mathcal{H}_{\varphi}}
\end{array}\right) \mid a^{\prime}, b^{\prime}, c^{\prime}, d^{\prime} \in \mathcal{M}^{\prime}\right\} .
$$

The proof is similar to that of [6, Proposition 1.2.2], so we omit the details. 
Next, we set

$$
J=\left(\begin{array}{cccc}
J_{\varphi} & 0 & 0 & 0 \\
0 & 0 & \left.J_{\varphi}\right|_{p^{\prime} \mathcal{H}_{\varphi}} & 0 \\
0 & \left.J_{\varphi}\right|_{p \mathcal{H}_{\varphi}} & 0 & 0 \\
0 & 0 & 0 & \left.J_{\varphi}\right|_{p p^{\prime} \mathcal{H}_{\varphi}}
\end{array}\right) .
$$

Our main purpose is to prove that $J_{\chi}=J$.

For simplicity, we write $\zeta^{\sharp}=\overline{S_{\chi}} \zeta, \zeta \in \mathcal{D}\left(\overline{S_{\chi}}\right)$ and $\eta^{b}=S_{\chi}^{*} \eta, \eta \in \mathcal{D}\left(S_{\chi}^{*}\right)$.

Lemma 4. (1) Suppose that $\zeta, \eta \in \mathfrak{B}_{\chi}$ satisfy

$$
\pi_{l}^{\chi}(\zeta)^{*}=\pi_{l}^{\chi}(\eta)
$$

Then $\zeta, \eta \in \mathfrak{A}_{\chi}$ and $\zeta^{\sharp}=\eta$.

(2) Suppose that $\zeta, \eta \in \mathfrak{B}_{\chi}^{\prime}$ satisfy

$$
\pi_{r}^{\chi}(\zeta)^{*}=\pi_{r}^{\chi}(\eta)^{*}
$$

Then $\zeta, \eta \in \mathfrak{A}_{\chi}^{\prime}$ and $\zeta^{b}=\eta$.

Proof. The proofs of (1) and (2) are similar, so we will prove only (1). Let $\zeta, \eta \in \mathfrak{B}_{\chi}$ satisfy $\pi_{l}^{\chi}(\zeta)^{*}=\pi_{l}^{\chi}(\eta)$, and let $\zeta^{\prime}, \eta^{\prime} \in \mathfrak{A}^{\prime}$. Then we have

$$
\begin{aligned}
\left(\zeta \mid\left(\zeta^{\prime} \eta^{\prime}\right)^{b}\right) & =\left(\zeta \mid \eta^{\prime b} \zeta^{\prime b}\right)=\left(\zeta \mid \pi_{r}^{\chi}\left(\zeta^{\prime}\right)^{*} \eta^{\prime b}\right)=\left(\pi_{r}^{\chi}\left(\zeta^{\prime}\right) \zeta \mid \eta^{\prime b}\right)=\left(\pi_{l}^{\chi}(\zeta) \zeta^{\prime} \mid \eta^{\prime b}\right) \\
& =\left(\zeta^{\prime} \mid \pi_{l}^{\chi}(\zeta)^{*} \eta^{\prime b}\right)=\left(\zeta^{\prime} \mid \pi_{l}^{\chi}(\eta) \eta^{\prime b}\right)=\left(\zeta^{\prime} \mid \pi_{r}^{\chi}\left(\eta^{\prime}\right)^{*} \eta\right) \\
& =\left(\pi_{r}^{\chi}\left(\eta^{\prime}\right) \zeta^{\prime} \mid \eta\right)=\left(\zeta^{\prime} \eta^{\prime} \mid \eta\right) .
\end{aligned}
$$

Since $\left(\mathfrak{A}_{\chi}^{\prime}\right)^{2}$ is a core for the ${ }^{b}$-operation ([11, Lemma VI.1.13]), and the ${ }^{b}$-operation and the ${ }^{\sharp}$-operation are mutually adjoint, we have $\zeta \in \mathcal{D}\left(\overline{S_{\chi}}\right)$ and $\zeta^{\sharp}=\eta$. Hence both $\zeta$ and $\eta$ belong to $\mathfrak{A}_{\chi}$.

Lemma 5. Suppose that $\eta \in \mathfrak{B}_{\chi}^{\prime}$ satisfies

$$
\pi_{r}^{\chi}(\eta)=\left(\begin{array}{cccc}
a^{\prime} & 0 & \left.b^{\prime}\right|_{p^{\prime} \mathcal{H}_{\varphi}} & 0 \\
0 & \left.a^{\prime}\right|_{p \mathcal{H}_{\varphi}} & 0 & \left.b^{\prime}\right|_{p p^{\prime} \mathcal{H}_{\varphi}} \\
p^{\prime} c^{\prime} & 0 & \left.p^{\prime} d^{\prime}\right|_{p^{\prime} \mathcal{H}_{\varphi}} & 0 \\
0 & \left.p^{\prime} c^{\prime}\right|_{p \mathcal{H}_{\varphi}} & 0 & \left.p^{\prime} d^{\prime}\right|_{p p^{\prime} \mathcal{H}_{\varphi}}
\end{array}\right) \in\left(\mathcal{N}_{\bar{p}}\right)^{\prime}
$$

for some $a^{\prime}, b^{\prime}, c^{\prime}, d^{\prime} \in \mathcal{M}^{\prime}$ (see Lemma 3). Then $\eta$ is of the form

$$
\eta=\left(\begin{array}{c}
\eta_{1} \\
b^{\prime} \xi \\
\eta_{3} \\
p^{\prime} d^{\prime} \xi
\end{array}\right)
$$

where $\eta_{1}, \eta_{3} \in \mathfrak{B}_{\varphi}^{\prime}, \pi_{r}\left(\eta_{1}\right)=a^{\prime}, \pi_{r}\left(\eta_{3}\right)=p^{\prime} c^{\prime}$.

Proof. Take

$$
\zeta=\left(\begin{array}{c}
\Lambda_{\varphi}(e) \\
\Lambda_{\varphi}(g) \\
f \xi \\
h \xi
\end{array}\right) \in \mathfrak{A}_{\chi}
$$


Then by Lemma 2, $e \in \mathfrak{n}_{\varphi} \cap \mathfrak{n}_{\varphi}^{*}$ and $f \in \mathfrak{n}_{\varphi}^{*} p$. Moreover, we have $\pi_{r}^{\chi}(\eta) \zeta=\pi_{l}^{\chi}(\zeta) \eta$ and

$$
\begin{aligned}
\pi_{r}^{\chi}(\eta) \zeta= & \left(\begin{array}{cccc}
a^{\prime} & 0 & \left.b^{\prime}\right|_{p^{\prime} \mathcal{H}_{\varphi}} & 0 \\
0 & \left.a^{\prime}\right|_{p \mathcal{H}_{\varphi}} & 0 & \left.b^{\prime}\right|_{p p^{\prime} \mathcal{H}_{\varphi}} \\
p^{\prime} c^{\prime} & 0 & \left.p^{\prime} d^{\prime}\right|_{p^{\prime} \mathcal{H}_{\varphi}} & 0 \\
0 & \left.p^{\prime} c^{\prime}\right|_{p \mathcal{H}_{\varphi}} & 0 & \left.p^{\prime} d^{\prime}\right|_{p p^{\prime} \mathcal{H}_{\varphi}}
\end{array}\right)\left(\begin{array}{c}
\Lambda_{\varphi}(e) \\
\Lambda_{\varphi}(g) \\
f \xi \\
h \xi
\end{array}\right) \\
= & \left(\begin{array}{c}
a^{\prime} \Lambda_{\varphi}(e)+b^{\prime} f \xi \\
a^{\prime} \Lambda_{\varphi}(g)+b^{\prime} h \xi \\
p^{\prime} c^{\prime} \Lambda_{\varphi}(e)+p^{\prime} d^{\prime} f \xi \\
p^{\prime} c^{\prime} \Lambda_{\varphi}(g)+p^{\prime} d^{\prime} h \xi
\end{array}\right),
\end{aligned}
$$

and putting $\eta=\left(\begin{array}{c}\eta_{1} \\ \eta_{2} \\ \eta_{3} \\ \eta_{4}\end{array}\right) \in \mathcal{H}_{\varphi} \oplus p \mathcal{H}_{\varphi} \oplus p^{\prime} \mathcal{H}_{\varphi} \oplus p p^{\prime} \mathcal{H}_{\varphi}$, we have

$$
\pi_{l}^{\chi}(\zeta) \eta=\left(\begin{array}{cccc}
e & \left.f\right|_{p \mathcal{H}_{\varphi}} & 0 & 0 \\
g & \left.h\right|_{p \mathcal{H}_{\varphi}} & 0 & 0 \\
0 & 0 & \left.e\right|_{p^{\prime} \mathcal{H}_{\varphi}} & \left.f\right|_{p p^{\prime} \mathcal{H}_{\varphi}} \\
0 & 0 & \left.g\right|_{p^{\prime} \mathcal{H}_{\varphi}} & \left.h\right|_{p p^{\prime} \mathcal{H}_{\varphi}}
\end{array}\right)\left(\begin{array}{l}
\eta_{1} \\
\eta_{2} \\
\eta_{3} \\
\eta_{4}
\end{array}\right)=\left(\begin{array}{c}
e \eta_{1}+f \eta_{2} \\
g \eta_{1}+h \eta_{2} \\
e \eta_{3}+f \eta_{4} \\
g \eta_{3}+h \eta_{4}
\end{array}\right)
$$

Hence we have

$$
\left(\begin{array}{c}
a^{\prime} \Lambda_{\varphi}(e)+b^{\prime} f \xi \\
a^{\prime} \Lambda_{\varphi}(g)+b^{\prime} h \xi \\
p^{\prime} c^{\prime} \Lambda_{\varphi}(e)+p^{\prime} d^{\prime} f \xi \\
p^{\prime} c^{\prime} \Lambda_{\varphi}(g)+p^{\prime} d^{\prime} h \xi
\end{array}\right)=\left(\begin{array}{c}
e \eta_{1}+f \eta_{2} \\
g \eta_{1}+h \eta_{2} \\
e \eta_{3}+f \eta_{4} \\
g \eta_{3}+h \eta_{4}
\end{array}\right)
$$

If we put $f=h=0$ in (2), we get

$$
a^{\prime} \Lambda_{\varphi}(e)=e \eta_{1}, p^{\prime} c^{\prime} \Lambda_{\varphi}(e)=e \eta_{3}
$$

for all $e \in \pi_{l}^{\chi}\left(\mathfrak{A}_{\varphi}\right)$. By the definition of $\mathfrak{B}_{\chi}^{\prime}$, we have $\eta_{1}, \eta_{3} \in \mathfrak{B}_{\chi}^{\prime}$ with

$$
\pi_{r}^{\chi}\left(\eta_{1}\right)=a^{\prime}, \pi_{r}^{\chi}\left(\eta_{3}\right)=p^{\prime} c^{\prime} .
$$

If we put $e=g=0$ in (2), we get

$$
f b^{\prime} \xi=f \eta_{2}, f p^{\prime} d^{\prime} \xi=f \eta_{4}
$$

for all $f \in \mathfrak{n}_{\varphi}^{*} p$. Since $p$ commutes with $b^{\prime}$ and $p^{\prime} d^{\prime}$, and $\eta_{2} \in p \mathcal{H}_{\varphi}, \eta_{4} \in p p^{\prime} \mathcal{H}_{\varphi},(3)$ holds also for all $f \in \mathfrak{n}_{\varphi}^{*}$. Since $\mathfrak{n}_{\varphi}^{*}\left(\supset \pi_{l}^{\chi}\left(\mathfrak{A}_{\varphi}\right)\right)$ is strongly dense in $\mathcal{M}$, we have

$$
\eta_{2}=b^{\prime} \xi, \eta_{4}=p^{\prime} d^{\prime} \xi \text {. }
$$

Hence we completed the proof.

Lemma 6. $J \mathfrak{A}_{\chi}=\mathfrak{A}_{\chi}^{\prime}$.

Proof. We first show that $J \mathfrak{A}_{\chi} \subset \mathfrak{A}_{\chi}^{\prime}$.

Let

We claim that $J \eta_{1} \in \mathcal{B}_{\chi}^{\prime}$.

$$
\eta_{1}=\left(\begin{array}{c}
\Lambda_{\varphi}(a) \\
\Lambda_{\varphi}(c) \\
b \xi \\
d \xi
\end{array}\right) \in \mathfrak{A}_{\chi}, \eta_{2}=\left(\begin{array}{c}
\Lambda_{\varphi}(e) \\
\Lambda_{\varphi}(g) \\
f \xi \\
h \xi
\end{array}\right) \in \mathfrak{A}_{\chi}
$$


We have

$$
\begin{aligned}
& \pi_{l}^{\chi}\left(\eta_{2}\right) J \eta_{1}=\pi_{l}^{\chi}\left(\left(\begin{array}{c}
\Lambda_{\varphi}(e) \\
\Lambda_{\varphi}(g) \\
f \xi \\
h \xi
\end{array}\right)\right)\left(\begin{array}{c}
J_{\varphi} \Lambda_{\varphi}(a) \\
J_{\varphi} b \xi \\
J_{\varphi} \Lambda_{\varphi}(c) \\
J_{\varphi} d \xi
\end{array}\right) \\
& =\left(\begin{array}{cccc}
e & \left.f\right|_{p \mathcal{H}_{\varphi}} & 0 & 0 \\
g & \left.h\right|_{p \mathcal{H}_{\varphi}} & 0 & 0 \\
0 & 0 & \left.e\right|_{p^{\prime} \mathcal{H}_{\varphi}} & \left.f\right|_{p^{\prime} \mathcal{H}_{\varphi}} \\
0 & 0 & \left.g\right|_{p^{\prime} \mathcal{H}_{\varphi}} & \left.h\right|_{p p^{\prime} \mathcal{H}_{\varphi}}
\end{array}\right)\left(\begin{array}{c}
J_{\varphi} \Lambda_{\varphi}(a) \\
J_{\varphi} b \xi \\
J_{\varphi} \Lambda_{\varphi}(c) \\
J_{\varphi} d \xi
\end{array}\right) \\
& =\left(\begin{array}{l}
e J_{\varphi} \Lambda_{\varphi}(a)+f J_{\varphi} b \xi \\
g J_{\varphi} \Lambda_{\varphi}(a)+h J_{\varphi} b \xi \\
e J_{\varphi} \Lambda_{\varphi}(c)+f J_{\varphi} d \xi \\
g J_{\varphi} \Lambda_{\varphi}(c)+h J_{\varphi} d \xi
\end{array}\right) .
\end{aligned}
$$

On the other hand,

$$
\begin{aligned}
& J \pi_{l}^{\chi}\left(\eta_{1}\right) J \eta_{2} \\
& =\left(\begin{array}{cccc}
J_{\varphi} & 0 & 0 & 0 \\
0 & 0 & \left.J_{\varphi}\right|_{p^{\prime} \mathcal{H}_{\varphi}} & 0 \\
0 & \left.J_{\varphi}\right|_{p \mathcal{H}_{\varphi}} & 0 & 0 \\
0 & 0 & 0 & \left.J_{\varphi}\right|_{p p^{\prime} \mathcal{H}_{\varphi}}
\end{array}\right) \pi_{l}^{\chi}\left(\left(\begin{array}{c}
\Lambda_{\varphi}(a) \\
\Lambda_{\varphi}(c) \\
b \xi \\
d \xi
\end{array}\right)\right) \\
& \times\left(\begin{array}{cccc}
J_{\varphi} & 0 & 0 & 0 \\
0 & 0 & \left.J_{\varphi}\right|_{p^{\prime} \mathcal{H}_{\varphi}} & 0 \\
0 & \left.J_{\varphi}\right|_{p \mathcal{H}_{\varphi}} & 0 & 0 \\
0 & 0 & 0 & \left.J_{\varphi}\right|_{p p^{\prime} \mathcal{H}_{\varphi}}
\end{array}\right)\left(\begin{array}{c}
\Lambda_{\varphi}(e) \\
\Lambda_{\varphi}(g) \\
f \xi \\
h \xi
\end{array}\right) \\
& =\left(\begin{array}{cccc}
J_{\varphi} a J_{\varphi} & 0 & \left.J_{\varphi} b J_{\varphi}\right|_{p^{\prime} \mathcal{H}_{\varphi}} & 0 \\
0 & \left.J_{\varphi} a J_{\varphi}\right|_{p \mathcal{H}_{\varphi}} & 0 & \left.J_{\varphi} b J_{\varphi}\right|_{p p^{\prime} \mathcal{H}_{\varphi}} \\
J_{\varphi} c J_{\varphi} & 0 & \left.J_{\varphi} d J_{\varphi}\right|_{p^{\prime} \mathcal{H}_{\varphi}} & 0 \\
0 & \left.J_{\varphi} c J_{\varphi}\right|_{p \mathcal{H}_{\varphi}} & 0 & \left.J_{\varphi} d v J_{\varphi}\right|_{p p^{\prime} \mathcal{H}_{\varphi}}
\end{array}\right)\left(\begin{array}{c}
\Lambda_{\varphi}(e) \\
\Lambda_{\varphi}(g) \\
f \xi \\
h \xi
\end{array}\right) \\
& =\left(\begin{array}{l}
J_{\varphi} a J_{\varphi} \Lambda_{\varphi}(e)+J_{\varphi} b J_{\varphi} f \xi \\
J_{\varphi} a J_{\varphi} \Lambda_{\varphi}(g)+J_{\varphi} b J_{\varphi} h \xi \\
J_{\varphi} c J_{\varphi} \Lambda_{\varphi}(e)+J_{\varphi} d J_{\varphi} f \xi \\
J_{\varphi} c J_{\varphi} \Lambda_{\varphi}(g)+J_{\varphi} d J_{\varphi} h \xi
\end{array}\right)=\left(\begin{array}{c}
e J_{\varphi} \Lambda_{\varphi}(a)+f J_{\varphi} b \xi \\
g J_{\varphi} \Lambda_{\varphi}(a)+h J_{\varphi} b \xi \\
e J_{\varphi} \Lambda_{\varphi}(c)+f J_{\varphi} d \xi \\
g J_{\varphi} \Lambda_{\varphi}(c)+h J_{\varphi} d \xi
\end{array}\right) .
\end{aligned}
$$

Hence we have $\pi_{l}^{\chi}\left(\eta_{2}\right) J \eta_{1}=J \pi_{l}^{\chi}\left(\eta_{1}\right) J \eta_{2}$. Consequently, we have

$$
J \eta_{1} \in \mathfrak{B}_{\chi}^{\prime} \text { and } \pi_{r}^{\chi}\left(J \eta_{1}\right)=J \pi_{l}^{\chi}\left(\eta_{1}\right) J
$$

Replacing $\eta_{1}$ by $\eta_{1}^{\sharp}$, we have

$$
J \eta_{1}^{\sharp} \in \mathfrak{B}_{\chi}^{\prime} \text { and } \pi_{r}^{\chi}\left(J \eta_{1}^{\sharp}\right)=J \pi_{l}^{\chi}\left(\eta_{1}^{\sharp}\right) J=\left(J \pi_{l}^{\chi}\left(\eta_{1}\right) J\right)^{*} .
$$

By Lemma $4(2), J \eta_{1} \in \mathfrak{A}_{\chi}^{\prime}$ with $\left(J \eta_{1}\right)^{b}=J \eta_{1}^{\sharp}$. Hence $J \mathfrak{A}_{\chi} \subset \mathfrak{A}_{\chi}^{\prime}$

Next, we will show that $\mathfrak{A}_{\chi} \supset J \mathfrak{A}_{\chi}^{\prime}$.

Take $\eta \in \mathfrak{A}_{\chi}^{\prime}$. By Lemma 5, $\eta$ is of the form

$$
\eta=\left(\begin{array}{c}
\eta_{1} \\
b^{\prime} \xi \\
\eta_{3} \\
d^{\prime} \xi
\end{array}\right),
$$


where $\eta_{1}, \eta_{3} \in \mathfrak{B}_{\varphi}^{\prime}$ and $b^{\prime}, d^{\prime} \in \mathcal{M}^{\prime}$ with

$$
\pi_{r}^{\chi}(\eta)=\left(\begin{array}{cccc}
\pi_{r}^{\varphi}\left(\eta_{1}\right) & 0 & \left.b^{\prime}\right|_{p^{\prime} \mathcal{H}_{\varphi}} & 0 \\
0 & \left.\pi_{r}^{\varphi}\left(\eta_{1}\right)\right|_{p \mathcal{H}_{\varphi}} & 0 & \left.b^{\prime}\right|_{p p^{\prime} \mathcal{H}_{\varphi}} \\
p^{\prime} \pi_{r}^{\varphi}\left(\eta_{3}\right) & 0 & \left.p^{\prime} d^{\prime}\right|_{p^{\prime} \mathcal{H}_{\varphi}} & 0 \\
0 & \left.p^{\prime} \pi_{r}^{\varphi}\left(\eta_{3}\right)\right|_{p \mathcal{H}_{\varphi}} & 0 & \left.p^{\prime} d^{\prime}\right|_{p p^{\prime} \mathcal{H}_{\varphi}}
\end{array}\right) .
$$

We claim that $J \eta \in \mathfrak{B}_{\chi}$. Since $J_{\varphi} \xi=\xi \in \mathcal{P}_{\varphi}^{\natural}$, we have

$$
J \eta=\left(\begin{array}{c}
J_{\varphi} \eta_{1} \\
J_{\varphi} \eta_{3} \\
J_{\varphi} b^{\prime} \xi \\
J_{\varphi} d^{\prime} \xi
\end{array}\right)=\left(\begin{array}{c}
J_{\varphi} \eta_{1} \\
J_{\varphi} \eta_{3} \\
b \xi \\
d \xi
\end{array}\right)
$$

where $b=J_{\varphi} b^{\prime} J_{\varphi} \in \mathcal{M}$ and $d=J_{\varphi} d^{\prime} J_{\varphi} \in \mathcal{M}$. Since $J_{\varphi} \eta_{1}, J_{\varphi} \eta_{3} \in J_{\varphi} \mathfrak{B}_{\varphi}^{\prime}=\mathfrak{B}_{\varphi}$, we have $J \eta \in \mathfrak{B}_{\chi}$ with

$$
\pi_{l}^{\chi}(J \eta)=\left(\begin{array}{cccc}
\pi_{l}^{\varphi}\left(J_{\varphi} \eta_{1}\right) & \left.b\right|_{p \mathcal{H}_{\varphi}} & 0 & 0 \\
\pi_{l}^{\varphi}\left(J_{\varphi} \eta_{3}\right) & \left.d\right|_{p \mathcal{H}_{\varphi}} & 0 & 0 \\
0 & 0 & \left.\pi_{l}^{\varphi}\left(J_{\varphi} \eta_{1}\right)\right|_{p^{\prime} \mathcal{H}_{\varphi}} & \left.b\right|_{p_{p^{\prime} \mathcal{H}_{\varphi}}} \\
0 & 0 & \left.\pi_{l}^{\varphi}\left(J_{\varphi} \eta_{3}\right)\right|_{p^{\prime} \mathcal{H}_{\varphi}} & \left.d\right|_{p p^{\prime} \mathcal{H}_{\varphi}}
\end{array}\right) .
$$

On the other hand,

$$
\begin{aligned}
J \pi_{r}^{\chi}(\eta) J & =\left(\begin{array}{cccc}
J_{\varphi} \pi_{r}^{\varphi}\left(\eta_{1}\right) J_{\varphi} & \left.J_{\varphi} b^{\prime} J_{\varphi}\right|_{p \mathcal{H}_{\varphi}} & 0 & 0 \\
J_{\varphi} \pi_{r}^{\varphi}\left(\eta_{3}\right) J_{\varphi} & \left.J_{\varphi} d^{\prime} J_{\varphi}\right|_{p \mathcal{H}_{\varphi}} & 0 & 0 \\
0 & 0 & \left.J_{\varphi} \pi_{r}^{\varphi}\left(\eta_{1}\right) J_{\varphi}\right|_{p^{\prime} \mathcal{H}_{\varphi}} & \left.J_{\varphi} b^{\prime} J_{\varphi}\right|_{p p^{\prime} \mathcal{H}_{\varphi}} \\
0 & 0 & \left.J_{\varphi} \pi_{r}^{\varphi}\left(\eta_{3}\right) J_{\varphi}\right|_{p^{\prime} \mathcal{H}_{\varphi}} & \left.J_{\varphi} d^{\prime} J_{\varphi}\right|_{p p^{\prime} \mathcal{H}_{\varphi}}
\end{array}\right) \\
& =\pi_{l}^{\chi}(J \eta) .
\end{aligned}
$$

Replacing $\eta$ by $\eta^{\text {b }}$, we have, $J \eta^{b} \in \mathfrak{B}_{\chi}$ with

$$
\pi_{l}^{\chi}\left(J \eta^{b}\right)=J \pi_{r}^{\chi}\left(\eta^{b}\right) J=\left(J \pi_{r}^{\chi}(\eta) J\right)^{*} .
$$

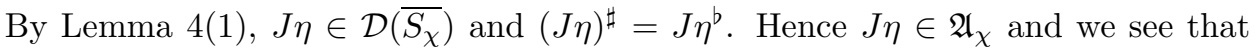
$J \mathfrak{A}_{\chi}^{\prime} \subset \mathfrak{A}_{\chi}$.

We have reached the main theorem, whose proof is a modification of $[1$, Theorem 1].

Theorem 7. $J=J_{\chi}$.

Proof. First, for

$$
s=\left(\begin{array}{ll}
a & b \\
c & d
\end{array}\right) \in \mathfrak{n}_{\chi} \cap \mathfrak{n}_{\chi}^{*}
$$

with

$$
\eta_{\chi}(s)=\left(\begin{array}{c}
\Lambda_{\varphi}(a) \\
\Lambda_{\varphi}(c) \\
b \xi \\
d \xi
\end{array}\right) \in \mathfrak{A}_{\chi}
$$


we have

$$
\begin{aligned}
& \left.\left(\eta_{\chi}\left(s^{*}\right) \mid J \eta_{\chi}(s)\right)=\left(\left(\begin{array}{c}
\Lambda_{\varphi}(a) \\
\Lambda_{\varphi}(c) \\
b \xi \\
d \xi
\end{array}\right)\right) J\left(\begin{array}{c}
\Lambda_{\varphi}(a) \\
\Lambda_{\varphi}(c) \\
b \xi \\
d \xi
\end{array}\right)\right) \\
& =\left(\left(\begin{array}{c}
\Lambda_{\varphi}\left(a^{*}\right) \\
\Lambda_{\varphi}\left(b^{*}\right) \\
c^{*} \xi \\
d^{*} \xi
\end{array}\right) \mid\left(\begin{array}{c}
J_{\varphi} \Lambda_{\varphi}(a) \\
J_{\varphi} b \xi \\
J_{\varphi} \Lambda_{\varphi}(c) \\
J_{\varphi} d \xi
\end{array}\right)\right) \\
& =\left(\Lambda_{\varphi}\left(a^{*}\right) \mid J_{\varphi} \Lambda_{\varphi}(a)\right)+\left(\Lambda_{\varphi}\left(b^{*}\right) \mid J_{\varphi} b \xi\right)+\left(c^{*} \xi \mid J_{\varphi} \Lambda_{\varphi}(c)\right)+\left(d^{*} \xi \mid J_{\varphi} d \xi\right) \\
& =\left(\Delta_{\varphi}^{1 / 2} \Lambda_{\varphi}(a) \mid \Lambda_{\varphi}(a)\right)+\overline{\left(b^{*} J_{\varphi} \Lambda_{\varphi}\left(b^{*}\right) \mid \xi\right)}+\left(\xi \mid c J_{\varphi} \Lambda_{\varphi}(c)\right)+\left(\xi \mid d J_{\varphi} d \xi\right) .
\end{aligned}
$$

Since $\xi, b^{*} J_{\varphi} \Lambda_{\varphi}\left(b^{*}\right), c J_{\varphi} \Lambda_{\varphi}(c)$ and $d J_{\varphi} d \xi$ all belong to $\mathcal{P}_{\varphi}^{\natural}$, we conclude that

$$
\left(\eta_{\chi}\left(s^{*}\right) \mid J \eta_{\chi}(s)\right) \geq 0
$$

Now, we define

$$
T \eta_{\chi}(s)=J \eta_{\chi}\left(s^{*}\right), s \in \mathfrak{n}_{\chi} \cap \mathfrak{n}_{\chi}^{*} .
$$

By the above calculations, we find that $T$ is positive symmetric, densely defined linear operator. It is readily seen that

$$
T=J S_{\chi}
$$

Since $J$ preserves the norm, we have

$$
\bar{T}=J \overline{S_{\chi}}
$$

and $\mathcal{D}(\bar{T})=\mathcal{D}\left(\overline{S_{\chi}}\right)=\mathcal{D}\left(\Delta_{\chi}^{1 / 2}\right)$. We will show that $\bar{T}$ is self-adjoint (then $\bar{T}$ is positive self-adjoint). Since $T$ is symmetric, we have $\bar{T} \subset T^{*}$. Since

$$
T^{*}=S_{\chi}^{*} J
$$

$\mathfrak{A}_{\chi}^{\prime}$ is a core for $S_{\chi}^{*}$ and since $J \mathfrak{A}_{\chi}=\mathfrak{A}_{\chi}^{\prime}$ (by Lemma 6 ), we see that $\mathfrak{A}_{\chi}$ is a core for $T^{*}$. On the other hand, $\mathfrak{A}_{\chi}$ is a core also for $\bar{T}$. Hence $\bar{T}=T^{*}$. Hence $\bar{T}$ and $\Delta_{\chi}^{1 / 2}$ are both positive self-adjoint. Then we have

$$
\bar{T}^{2}=T^{*} \bar{T}=S_{\chi}^{*} J J \overline{S_{\chi}}=\Delta_{\chi} .
$$

By the uniqueness of the positive square root, we have

$$
\bar{T}=\Delta_{\chi}^{1 / 2}
$$

On the other hand, $\bar{T}=J J_{\chi} \Delta_{\chi}^{1 / 2}$. Hence we have $J=J_{\chi}$.

\section{REFERENCES}

[1] H. Araki, Some properties of modular conjugation operator of von Neumann algebras and a non-commutative Radon-Nikodym theorem with a chain rule., Pacific J. Math. 50 (1974), 309-354. MR 50:2929

[2] A. Connes, Caractérisation des espaces vectoriels ordonnés sous-jacent aux algèbres de von Neumann, Ann. Inst. Fourier 24 (1974), 121-155. MR 51:13705

[3] T. Digernes, Duality for weights on covariant systems and its applications, Dissertation, Los Angeles, 1975.

[4] U. Haagerup, The standard form of von Neumann algebras, Math. Scand. 37 (1975), 271-283. MR 53:11387

[5] H. Izumi, Natural bilinear forms, natural sesquilinear forms and the associated duality of non-commutative $L^{p}$-spaces, to appear in Int. J. Math. 
[6] H. Kosaki, Canonical L ${ }^{p}$-Spaces Associated with an Arbitrary Abstract von Neumann Algebra, Thesis, UCLA, 1980.

[7] H. Kosaki, Applications of the Complex Interpolation Method to a von Neumann Algebra: Non-commutative $L^{p}$-Spaces, J. Funct. Anal. 56 (1984), 29-78. MR 86a:46085

[8] S. Strătilă, Modular Theory in Operator Algebras, Abacus Press, 1981. MR 85g:46072

[9] M. Takesaki, Theory of Operator Algebras I, Springer-Verlag, 1979. MR 81e:46038

[10] M. Takesaki, The structure of operator algebras (in Japanese), Iwanami Shoten, 1983. MR 86f: 46062

[11] M. Takesaki, Theory of Operator Algebras II (to be published).

Mathematical Institute, Tohoku University, Aza-Aoba, Aramaki, Aoba-ku, Sendai 980-8578, JAPAN

E-mail address: h-izumi@math.tohoku.ac.jp 